\title{
The Scottish diet is poorer in households purchasing tobacco products: analysis of Living Costs and Food Survey data from 2001-2012
}

\author{
L.F. Masson ${ }^{1}$, K.L. Barton ${ }^{2}$ and W.L. Wrieden ${ }^{3}$ \\ ${ }^{1}$ School of Pharmacy and Life Sciences, Robert Gordon University, Aberdeen, AB10 7 GJ, ${ }^{2}$ Division of Food and Drink, \\ Abertay University, Dundee, DDI 1 HG and ${ }^{3}$ Human Nutrition Research Centre and Institute of Health and Society, \\ Newcastle University, Newcastle upon Tyne, NE2 $4 H H$
}

Research suggests that smokers report lower self-efficacy, overall motivation and intrinsic motivation for meeting dietary recommendations $^{(1)}$. This research aimed to assess whether smokers in Scotland have a poorer diet than non-smokers.

Household food purchase data for Scotland between 2001 and 2012 from the UK Living Costs and Food Survey was pooled to estimate intakes of fruit and vegetables, oily fish, total fat, saturated fatty acids (SFA), non-milk extrinsic sugars (NMES) and nonstarch polysaccharides (NSP) by category of tobacco purchases. Households making tobacco purchases were categorised into thirds of purchase (low, medium and high), with tertiles calculated on an annual basis. Adjustments were made for waste ${ }^{(2)}$ and data were analysed using general linear models within the complex samples module of SPSS (SPSS Inc., Chicago, IL, USA), weighting to the Scottish population and taking account of sampling methods. Dietary intakes were adjusted for Scottish Index of Multiple Deprivation quintile, and estimated marginal mean intakes with $95 \%$ confidence intervals are presented by category of tobacco purchases.

Compared with households that made no tobacco purchases, households that purchased tobacco had significantly lower intakes of fruit and vegetables, oily fish and NSP, and significantly higher intakes of total fat, SFA and NMES (data not shown). The table shows that there are significant associations between dietary intakes and categories of tobacco purchases. Dietary differences between groups were most pronounced for fruit and vegetables.

\begin{tabular}{|c|c|c|c|c|c|}
\hline & \multicolumn{4}{|c|}{ Tobacco purchases } & \multirow[b]{2}{*}{ overall association } \\
\hline & $\begin{array}{l}\text { None } \\
n 4499 \\
\text { wt } n 40497\end{array}$ & $\begin{array}{l}\text { Low } \\
n 647 \\
\text { wt } n 7178\end{array}$ & $\begin{array}{l}\text { Medium } \\
n 657 \\
\text { wt } n 7092\end{array}$ & $\begin{array}{l}\text { High } \\
n 652 \\
\text { wt } n 5652\end{array}$ & \\
\hline Fruit \& vegetables ${ }^{1}$ (g/day) & $299[291,307]$ & $232[215,248]$ & $210[194,225]$ & $200[189,211]$ & $<0.001$ \\
\hline Oily fish (g/week) & $34.7[31 \cdot 7,37 \cdot 6]$ & $23.9[20 \cdot 7,27 \cdot 2]$ & $21 \cdot 1[17 \cdot 2,24 \cdot 9]$ & $22 \cdot 6[17 \cdot 3,28 \cdot 0]$ & $<0.001$ \\
\hline Total fat ( $\%$ energy) & $38.4[38 \cdot 1,38 \cdot 6]$ & $39.4[38 \cdot 8,40 \cdot 0]$ & $39.2[38.6,39.8]$ & $40 \cdot 3[39 \cdot 7,40 \cdot 9]$ & $<0 \cdot 001$ \\
\hline SFA ( \% energy) & $15 \cdot 1[15 \cdot 0,15 \cdot 3]$ & $15 \cdot 1[14 \cdot 9,15 \cdot 4]$ & $15 \cdot 4[15 \cdot 2,15 \cdot 6]$ & $15 \cdot 8[15 \cdot 5,16 \cdot 0]$ & $<0.001$ \\
\hline NMES (\% energy) & $15 \cdot 0[14 \cdot 8,15 \cdot 2]$ & $15.3[14.8,15.9]$ & $15.9[15 \cdot 2,16 \cdot 6]$ & $15 \cdot 6[14.9,16 \cdot 2]$ & $0 \cdot 012$ \\
\hline NSP (g/day) & $12 \cdot 9[12 \cdot 7,13 \cdot 2]$ & $11.4[10 \cdot 8,12 \cdot 0]$ & $11 \cdot 3[10 \cdot 8,11 \cdot 8]$ & $11.4[10 \cdot 9,12 \cdot 0]$ & $<0.001$ \\
\hline
\end{tabular}

$n$, number of households; $w t n$, weighted number of people in the sample

${ }^{1}$ Includes fruit and vegetable juice and baked beans

Results suggest that smokers have a poorer diet that non-smokers, and the diet is poorer in heavier smokers than light smokers. This diet-smoking association is independent of deprivation level. Smokers in Scotland are an important target group for dietary intervention.

Funded by Food Standards Scotland, project FS424018. Data from DEFRA, SNS, ONS and the UK Data Archive.

1. McClure JB, Divine G, Alexander G et al. (2009). Behav Med 35, 14-22.

2. Waste and Resource Action Programme (2007). The food we waste. Oxon: WRAP 